\title{
Which Clinical and Patient Factors Influence the National Economic Burden of Hospital Readmissions After Total Joint Arthroplasty?
}

\author{
Steven M. Kurtz PhD, Edmund C. Lau MS, Kevin L. Ong PhD, Edward M. Adler MD, \\ Frank R. Kolisek MD, Michael T. Manley FRSA, PhD
}

Published online: 20 January 2017

(C) The Association of Bone and Joint Surgeons@ 2017

\begin{abstract}
Background The Affordable Care Act of 2010 advanced the economic model of bundled payments for total joint arthroplasty (TJA), in which hospitals will be financially responsible for readmissions, typically at 90 days after surgery. However, little is known about the financial burden of readmissions and what patient, clinical, and hospital factors drive readmission costs.

Questions/purposes (1) What is the incidence, payer mix, and demographics of THA and TKA readmissions in the United States? (2) What patient, clinical, and hospital factors are associated with the cost of 30- and 90-day readmissions after primary THA and TKA? (3) Are there
\end{abstract}

This study was supported by a research grant from Stryker Orthopaedics (SMK, Principal Investigator; Mahwah, NJ, USA). All ICMJE Conflict of Interest Forms for authors and Clinical Orthopaedics and Related Research ${ }^{\mathbb{R}}$ editors and board members are on file with the publication and can be viewed on request.

Each author certifies that his institution approved the human protocol for this investigation and that the investigation was conducted in conformity with ethical principles of research.

This work was performed at Exponent, Inc, Philadelphia, PA, USA.

Electronic supplementary material The online version of this article (doi:10.1007/s11999-017-5244-6) contains supplementary material, which is available to authorized users.

S. M. Kurtz ( $\square)$, K. L. Ong

Exponent Inc, 3440 Market Street, Suite 600, Philadelphia, PA

19104, USA

e-mail: skurtz@exponent.com

S. M. Kurtz

School of Biomedical Engineering, Science, and Health

Systems, Drexel University, Philadelphia, PA, USA

E. C. Lau

Exponent Inc, Menlo Park, CA, USA any differences in the economic burden of THA and TKA readmissions between payers? (4) What types of THA and TKA readmissions are most costly to the US hospital system?

Methods The recently developed Nationwide Readmissions Database from the Healthcare Cost and Utilization Project (2006 hospitals from 21 states) was used to identify 719,394 primary TJAs and 62,493 90-day readmissions in the first 9 months of 2013 based on International Classification of Diseases, 9th Revision, Clinical Modification codes. We classified the reasons for readmissions as either procedure- or medical-related. Cost-to-charge ratios supplied with the Nationwide Readmissions Database were used to compute the individual per-patient cost of 90-day readmissions as a continuous variable in separate general linear models for THA and TKA. Payer, patient, clinical, and hospital factors were treated as covariates. We estimated the national burden of readmissions by payer and by the reason for readmission.

Results The national rates of 30- and 90-day readmissions after THA were $4 \%$ (95\% confidence interval [CI], 4.2\%$4.5 \%)$ and $8 \%$ (95\% CI, 7.5\%-8.1\%), respectively. The national rates of 30- and 90-day readmissions after primary TKA were $4 \%(95 \% \mathrm{CI}, 3.8 \%-4.0 \%)$ and $7 \%(95 \% \mathrm{CI}$, $6.8 \%-7.2 \%)$, respectively. The five most important

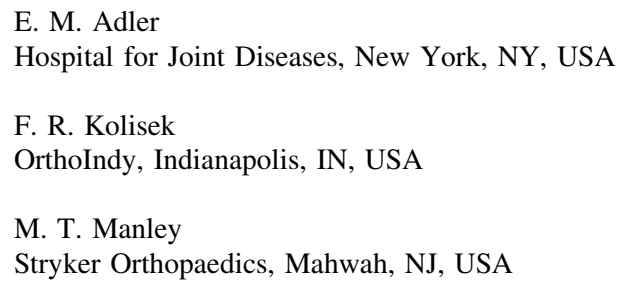


variables responsible for the cost of 90-day THA readmissions (in rank order, based on the Type III F-statistic, $\mathrm{p}<0.001$ ) were length of stay (LOS), all patient-refined diagnosis-related group (APR DRG) severity, type of readmission (that is, medical- versus procedure-related), hospital ownership, and age. Likewise, the five most important variables responsible for the cost of 90-day TKA readmissions were LOS, APR DRG severity, gender, hospital procedure volume, and hospital ownership. After adjusting for covariates, mean 90-day readmission costs reimbursed by private insurance were, on average, USD 1324 and USD 1372 greater than Medicare $(p<0.001)$ for THA and TKA, respectively. In the 90 days after TJA, two-thirds of the total annual readmission costs were covered by Medicare. In 90 days after THA, more readmissions were still associated with procedure-related complications, including infections, dislocations, and periprosthetic fractures, which in aggregate account for $59 \%(95 \%$ CI, $59.1 \%-59.6 \%)$ of the total readmission costs to the US healthcare system. For TKA, $49 \%$ of the total readmission cost (95\% CI, 48.8\%-49.6\%) in 90 days for the United States was associated with procedure issues, most notably including infections.

Conclusions Hospital readmissions up to 90 days after TJA represent a massive economic burden on the US healthcare system. Approximately half of the total annual economic burden for readmissions in the United States is medical and unrelated to the joint replacement procedure and half is related to procedural complications.

Clinical Relevance This national study underscores LOS during readmission as a primary cost driver, suggesting that hospitals and doctors further optimize, to the extent possible, the clinical pathways for the hospitalization of readmitted patients. Because patients readmitted as a result of infection, dislocation, and periprosthetic fractures are the most costly types of readmissions, efforts to reduce the LOS for these types of readmissions will have the greatest impact on their economic burden. Additional clinical research is needed to determine the extent to which, if any, the LOS during readmissions can be reduced without sacrificing quality or access of care.

\section{Introduction}

The national health expenditure in the United States is expected to increase to USD 5.4 trillion by 2024, at which point it will account for $20 \%$ of the gross domestic product [18]. With almost USD 1 out of every USD 5 generated by the US economy projected to be spent on health care, it is little wonder that policymakers and legislators have focused attention on limiting the growth of these obligations to a sustainable level, resulting in the megatrend of cost containment that health care in general [13, 20, 28, 39], and orthopaedics in particular [5, 7, 15], has been operating within for over a decade. Currently more than a million joint replacements are performed annually in the United States. As a result, orthopaedic procedures such as THA and TKA exert considerable leverage on national healthcare spending [23].

The challenge with healthcare reform has been to reduce spending without sacrificing quality in the delivery of care [4, 12, 19]. Not surprisingly, the Centers for Medicare \& Medicaid Services (CMS) under the framework of the Affordable Care Act of 2010 (ACA) has specifically identified THA and TKA for implementation of new quality benchmarks and innovative economic models such as bundled payments in an effort to address this cost issue $[5,14]$. Integral to these reforms, hospital readmissions have emerged in the ACA as a key quality metric and are formalized in the Medicare Hospital Readmissions Reduction Program (HRRP) [2, 16, 35]. An underlying tenet of recent healthcare legislation is that, for a period ranging between 30 and 90 days after discharge after THA or TKA, hospitals and/or Accountable Care Organizations (ACOs) will be financially responsible for readmissions for any reason. Healthcare policymakers euphemistically refer to this innovative strategy as "risk-sharing." On the upside, hospitals and ACOs are in a position to financially profit when their care delivery pathways minimize complications and unplanned readmissions. These new policies of the HRRP expose hospitals and ACOs to financial risk with penalties that range from $1 \%$ of $3 \%$ of their aggregate payments in a single year based on their excess 30-day readmissions. Researchers from a high-volume, urban tertiary orthopaedic center [9] recently estimated that their potential penalty from CMS could amount to over USD 6 million annually if their institution's all-cause risk-adjusted 30-day readmission rates exceeded the national mean. Healthcare bundle providers are exposed to the additional financial risk of accountability for the cost of readmissions during the bundle period (30 or 90 days) regardless of whether the readmission is causally related to the index surgery. Policymakers have crafted a carrot and two-stick approach to hospital readmissions: one stick is wielded by the HRRP and a second by the bundle payer.

Many researchers have studied the risk for readmissions after primary total joint arthroplasty [1, 10, 11, 17, 21, 22, 24, 25, 27, 29, 32, 33, 36-38]. However, less is known about the financial impact of total joint readmissions. The scope of previous economic studies has been limited to a single institution [3, 8, 9, 31, 34]; a proprietary commercial database [26]; or the state of Michigan [6]. The recent release of the novel Nationwide Readmissions Database (NRD) by the Agency for Healthcare Research and Quality (AHRQ) for all age groups and payers prompted us to 
examine the economic burden of total joint readmissions from a national perspective.

We used the NRD to address the following research questions: (1) What is the incidence, payer mix, and demographics of THA and TKA readmissions in the United States? (2) What patient, clinical, and hospital factors are associated with the cost of 30- and 90-day readmissions after primary THA and TKA? (3) Are there any differences in the economic burden of THA and TKA readmissions between payers? (4) What types of THA and TKA readmissions are most costly to the US hospital system?

\section{Materials and Methods}

The NRD from the Healthcare Cost and Utilization Project (HCUP) was used in this study. The NRD is a newly created database drawn from the HCUP State Inpatient Database (SID) program with verified patient linkage numbers that can be used to track readmissions across hospitals within a state. The NRD includes discharges from community hospitals, excluding rehabilitation or long-term acute care hospitals, from 21 participating partner states that contribute discharge data to the program. These states came from all regions of the United States and account for $49 \%$ of the total US resident population and $49 \%$ of all US hospitalizations. The first release of the NRD is for hospital discharges in 2013 and contained approximately 14 million records from 2006 hospitals. The HCUP calculated discharge weights for each NRD record based on the patient and hospital stratum in the SID data to represent 36 million discharges for the US population. Because the records are not linked across states, only in-state readmissions can be identified.

The NRD is the only nationally representative database that is dedicated to the study of hospital readmissions. Actual dates were removed from the original records. An arbitrary index date was assigned to each individual and each hospital admission is translated into relative-days from this arbitrary index. For example, if THA was recorded on "date" 10,000 and another hospital admission on "date" 10,045 , then this patient had a readmission 45 days after the THA. The strength of the database is its coverage of patients of all ages and payers (eg, Medicare, private insurance as well as the uninsured). The NRD was designed by the AHRQ specifically to inform national health policy regarding hospital readmissions (https:// www.hcup-us.ahrq.gov/nrdoverview.jsp).

Patients undergoing primary THA and TKA were identified in the NRD using International Classification of Diseases, 9th Revision, Clinical Modification (ICD-9-CM) procedure codes 81.51 and 81.54 , respectively. The timeframe of the analysis included any primary joint arthroplasties between January 1 and September 30, 2013, so that we would capture readmissions within 90 days of the 12-month period in which the NRD was active. Out-ofstate patients were excluded because, if readmission was necessary, these patients would likely receive treatment at a local facility rather than returning to the state where they received the primary arthroplasty. Additionally, a small fraction of patients who died at the hospital after the THA or TKA were also excluded. The out-of-state and dead patient exclusion criteria removed $6 \%$ of the initial sample size (from 1,056,661 to 991,899). Restricting the procedures to the first 9 months of the year resulted in a final sample size of 250,884 THAs and 468,510 TKAs.

We classified the reasons for readmissions at 30 and 90 days as either procedure- or medical-related based on the primary ICD-9 diagnosis code. We classified procedure-related readmissions to include: periprosthetic/ implant fractures (996.43, 996.44); dislocations (996.42); loosening (996.41); cellulitis/abscess of the leg (682.6); deep infections (996.66); septicemia (038.9); hematoma (998.1); acute anemia (285.1); acute cardiac events (410.x, 427.x, 428.x, 433.x 434.x) as well as acute vascular and thrombotic events $(415.1,453 . x)$. We identified 279 individual diagnosis codes that were classified as procedurerelated (Appendix 1 [Supplemental materials are available with the online version of $C O R R^{\circledR}$.]). Diagnoses not classified as procedure-related were classified as medicalrelated readmissions. Examples of medical-related readmissions, most likely unrelated to a primary joint replacement, included cancer, acute poisoning, psychiatric disorders, urologic and gastrointestinal diseases, and open fractures.

Cost-to-charge ratios supplied with the NRD were used to compute the hospital cost per patient of 30- and 90-day readmissions as a continuous variable in a general linear model in which the payer as well as patient, clinical, and hospital factors were treated as covariates. Patient factors we considered included: age; gender; all patient-refined diagnosis-related group (APR DRG) severity; number of comorbidities; total number of diagnoses coded; median income based on residence zip code; payer; and the population size of the patient's residence. We included the following clinical factors: the reason for readmission (that is, medical- or procedure-related) and length of stay. The following hospital factors were included in the analysis: hospital bed size, annual volume of hospital discharges, annual volume of total joint arthroplasty discharges, hospital location (rural, urban), ownership (private for profit, public, private nonprofit), and teaching status.

Each of the individual patient records in the NRD is accompanied by a weighting factor that was used to provide a national estimate of readmission cost as well as $95 \%$ confidence limits. We estimated the national cost of 
readmissions by payer and the reason for readmission. Because the timeframe of the study corresponded to a 9month observation window of primary surgeries, we obtained annualized national estimates by adjusting the NRD estimates by a factor of $4 / 3$.

Statistical analyses were performed using the SAS statistical software, Release 9.4 (Cary, NC, USA). Results were considered significant for statistical tests with $\mathrm{p}<0.05$. For many factors with the same significant $\mathrm{p}$ value (eg, < 0.0001 ), we examined the Type III tests of fixed effect measured by the F-statistics as an indicator for the relative importance of these hospital and patient factors on 30-day or 90-day readmission. The Type III F-statistics of a particular factor measure the additional reduction in the error variance after all the other factors had been included. It thus reflects the factor's independent contribution toward accounting for the variations in the dependent variable. The Type III F-statistic is valid for unbalanced data, which is typical in observational data sources used in such investigations.

\section{Results}

Incidence, Payer Mix, and Demographics of THA and TKA Readmissions in the United States

The national rates of 30- and 90-day readmissions after primary THA were $4 \%$ (95\% confidence interval [CI], $4.2 \%-4.5 \%)$ and $8 \%$ (95\% CI, 7.5\%-8.1\%), respectively. There were an estimated 250,884 primary THAs $(95 \%$ CI, 232,749-269,019) in the United States during the 9 months from January to September 2013 followed by 10,897 (95\% CI, 10,242-11,551) 30-day readmissions and 19,629 (95\% CI, 18,462-20,796) 90-day readmissions. Based on the number of cases, $69 \%$ of the 30-day readmissions and $67 \%$ of the 90-day readmissions for THA were reimbursed by Medicare (Table 1). In terms of demographics, readmitted patients who had undergone THA (90 days) were older (68 \pm 20 years) than the primary surgery recipients (65 \pm 18 years, $p<0.001)$, but the gender ratio was similar $(\mathrm{p}=0.312)($ Table 1$)$.

The national rates of 30- and 90-day readmissions after primary TKA were $4 \%(95 \% \mathrm{CI}, 3.8 \%-4.0 \%)$ and $7 \%$ (95\% CI, 6.8\%-7.2\%), respectively. There were 468,510 primary TKAs $(95 \%$ CI, 441,041-495,979) for the 9-month study period followed by 18,225 (95\% CI, 17,197-19,253) 30-day readmissions and 32,900 (95\% CI, 31,155-34,644) 90-day readmissions. Based on the number of cases, $68 \%$ of the 30-day readmissions and $66 \%$ of the 90-day readmissions for TKA were reimbursed by Medicare (Table 2). Readmitted patients who had undergone TKA (90 days) were older (68 \pm 17 years) than primary patients
$(66 \pm 15$ years, $\mathrm{p}<0.001)$. For patients who had undergone primary TKA, 63\% were female. For the readmitted patients, 59\% were female $(\mathrm{p}<0.001)$ (Table 2).

Patient, Clinical, and Hospital Factors and the Cost of Readmissions After Primary THA and TKA

The five most important variables responsible for the cost of 90-day THA readmissions (in rank order, based on the Type III F-statistic, $p<0.001$; Fig. 1) were length of stay (LOS), APR DRG severity, type of readmission (that is, medical- versus procedure-related), hospital ownership, and age. The mean $( \pm$ SD) cost per readmitted THA patient within 30 days was USD 15,895 \pm USD 317 and within 90 days was USD 18,207 \pm USD 294. The 90-day readmission cost was comparable to the cost of the primary surgery of USD 18,603 \pm USD 276.

The five most important variables responsible for the cost of 90-day TKA readmissions (in rank order, based on the Type III F-statistic, p < 0.001; Fig. 2) were LOS, APR DRG severity, gender, hospital procedure volume, and hospital ownership. The mean $( \pm \mathrm{SD})$ cost per readmitted patient who had undergone TKA within 30 days was USD $11,958 \pm$ USD 220 and within 90 days was USD $14,332 \pm$ USD 197; here the 90-day readmission cost was lower than the cost of the primary surgery, USD $17,715 \pm$ USD 255 .

Differences in the Economic Burden of THA and TKA Readmissions Between Payers

After adjusting for covariates, mean 90-day THA readmission costs reimbursed by private insurance were, on average, USD 1324 greater than Medicare $(\mathrm{p}<0.001)$ on a per-patient basis. Similarly, for TKA, after adjusting for covariates, mean 90-day readmission costs reimbursed by private insurance were, on average, USD 1372 greater than Medicare $(\mathrm{p}<0.001)$ on a per-patient basis.

In the 90 days after total joint arthroplasty, two-thirds of the total annual readmission costs were covered by Medicare. For the entire United States, Medicare covered USD 319 million per year, or $67 \%$ of the total annual cost for $90-$ day THA readmission (95\% CI, 66.6\%-67.4\%). The total annualized cost for 90-day readmission after THA in the United States was USD 477 million (95\% CI, USD 446507 million) with more than 171,000 days of hospital stays. For TKA, Medicare covered USD 417 million per year, or $66 \%$ of the total annual cost for 90 -day readmission $(95 \%$ CI, $65.8 \%-66.5 \%)$. The overall annualized total cost for 90-day readmission after TKA in the United States was USD 629 million (95\% CI, USD 593-664 million) with 
Table 1. Patients undergoing primary THA in the United States, January to September 2013, and associated 30- and 90-day readmissions from the National Readmission Database

\begin{tabular}{|c|c|c|c|}
\hline Selected patient, clinical, and hospital factors & Primary THAs & 30-day readmissions & 90-day readmissions \\
\hline Total & $250,884(100 \%)$ & $10,897(100 \%)$ & $19,629(100 \%)$ \\
\hline \multicolumn{4}{|l|}{ Sex } \\
\hline Male & $109,237(44 \%)$ & $4660(43 \%)$ & $8413(43 \%)$ \\
\hline Female & $141,647(56 \%)$ & $6236(57 \%)$ & $11,215(57 \%)$ \\
\hline \multicolumn{4}{|l|}{ Age group (years) } \\
\hline$<40$ & $5842(2 \%)$ & $215(2 \%)$ & $465(2 \%)$ \\
\hline $40-49$ & $16,682(7 \%)$ & $550(5 \%)$ & $1133(6 \%)$ \\
\hline $50-59$ & $54,994(22 \%)$ & $1803(17 \%)$ & $3486(18 \%)$ \\
\hline $60-69$ & $80,022(32 \%)$ & $2818(26 \%)$ & $5183(26 \%)$ \\
\hline $70-79$ & $62,967(25 \%)$ & $3107(29 \%)$ & $5286(27 \%)$ \\
\hline $80+$ & $30,377(12 \%)$ & $2405(22 \%)$ & $4076(21 \%)$ \\
\hline \multicolumn{4}{|l|}{ APR DRG } \\
\hline Minor & $120,918(48 \%)$ & $3630(33 \%)$ & $6898(35 \%)$ \\
\hline Moderate & $115,114(45 \%)$ & $5447(50 \%)$ & $9737(50 \%)$ \\
\hline Major & $13,640(5 \%)$ & $1572(14 \%)$ & $2618(13 \%)$ \\
\hline Extreme & $1211(0.5 \%)$ & $248(2 \%)$ & $376(2 \%)$ \\
\hline \multicolumn{4}{|l|}{ Income quartile } \\
\hline First & $47,465(19 \%)$ & $2504(23 \%)$ & $4405(22 \%)$ \\
\hline Second & $64,784(26 \%)$ & $2869(26 \%)$ & $5265(27 \%)$ \\
\hline Third & $67,450(27 \%)$ & $2836(26 \%)$ & $5186(26 \%)$ \\
\hline Fourth & $71,184(28 \%)$ & $2688(25 \%)$ & $4772(24 \%)$ \\
\hline \multicolumn{4}{|l|}{ Resident area } \\
\hline Metropolitan (large) & $121,813(49 \%)$ & $5354(49 \%)$ & $9564(49 \%)$ \\
\hline Metropolitan (medium) & $54,502(22 \%)$ & $2333(21 \%)$ & $4221(22 \%)$ \\
\hline Metropolitan (small) & $27,970(11 \%)$ & $1176(11 \%)$ & $2091(11 \%)$ \\
\hline Micropolitan & $27,401(11 \%)$ & $1216(11 \%)$ & $2229(11 \%)$ \\
\hline Rural & $19,152(8 \%)$ & $814(7 \%)$ & $1520(8 \%)$ \\
\hline \multicolumn{4}{|l|}{ Hospital size } \\
\hline Small & $48,930(20 \%)$ & $1864(17 \%)$ & $3316(17 \%)$ \\
\hline Medium & $60,803(24 \%)$ & $2600(24 \%)$ & $4602(23 \%)$ \\
\hline Large & $141,151(56 \%)$ & $6433(59 \%)$ & $11,711(60 \%)$ \\
\hline \multicolumn{4}{|l|}{ Hospital type } \\
\hline Public & $23,133(9 \%)$ & $1082(10 \%)$ & $1972(10 \%)$ \\
\hline Nonprofit & $196,793(78 \%)$ & $8082(74 \%)$ & $14,621(74 \%)$ \\
\hline Private & $30,958(12 \%)$ & $1733(16 \%)$ & $3035(15 \%)$ \\
\hline \multicolumn{4}{|l|}{ Teaching } \\
\hline Yes & $123,896(49 \%)$ & $5764(53 \%)$ & $10,299(52 \%)$ \\
\hline No & $126,988(51 \%)$ & $5132(47 \%)$ & $9329(48 \%)$ \\
\hline \multicolumn{4}{|l|}{ Payer } \\
\hline Medicare & $137,477(55 \%)$ & $7553(69 \%)$ & $13,180(67 \%)$ \\
\hline Private & $93,320(37 \%)$ & $2382(22 \%)$ & $4698(24 \%)$ \\
\hline Medicaid & $9828(4 \%)$ & $616(6 \%)$ & $1109(6 \%)$ \\
\hline Other & $10,259(4 \%)$ & $346(3 \%)$ & $642(3 \%)$ \\
\hline
\end{tabular}

APR DRG = all patient-refined diagnosis-related group. 
Table 2. Patients undergoing primary TKA in the United States, January to September 2013, and associated 30- and 90-day readmissions from the National Readmission Database

\begin{tabular}{|c|c|c|c|}
\hline Selected patient, clinical, and hospital factors & Primary TKAs & 30-day readmissions & 90-day readmissions \\
\hline Total & $468,510(100 \%)$ & $18,225(100 \%)$ & $32,900(100 \%)$ \\
\hline \multicolumn{4}{|l|}{$\operatorname{Sex}$} \\
\hline Male & $172,560(37 \%)$ & $7753(43 \%)$ & $13,432(41 \%)$ \\
\hline Female & $295,950(63 \%)$ & $10,472(57 \%)$ & $19,468(59 \%)$ \\
\hline \multicolumn{4}{|l|}{ Age group (years) } \\
\hline$<40$ & $2148(0.5 \%)$ & $161(1 \%)$ & $272(1 \%)$ \\
\hline $40-49$ & $19,555(4 \%)$ & $705(4 \%)$ & $1408(4 \%)$ \\
\hline $50-59$ & $94,851(20 \%)$ & $2952(16 \%)$ & $5792(18 \%)$ \\
\hline $60-69$ & $171,964(37 \%)$ & $5536(30 \%)$ & $10,477(32 \%)$ \\
\hline $70-79$ & $136,379(29 \%)$ & $5977(33 \%)$ & $10,235(31 \%)$ \\
\hline $80+$ & $43,613(9 \%)$ & $2895(16 \%)$ & $4717(14 \%)$ \\
\hline \multicolumn{4}{|l|}{ APR DRG } \\
\hline Minor & $227,941(49 \%)$ & $6802(37 \%)$ & $12,502(38 \%)$ \\
\hline Moderate & $215,858(46 \%)$ & $9213(51 \%)$ & $16,657(51 \%)$ \\
\hline Major & $23,421(5 \%)$ & $2003(11 \%)$ & $3405(10 \%)$ \\
\hline Extreme & $1286(0.3 \%)$ & $207(1 \%)$ & $336(1 \%)$ \\
\hline \multicolumn{4}{|l|}{ Income quartile } \\
\hline First & $97,818(21 \%)$ & $4421(24 \%)$ & $7835(24 \%)$ \\
\hline Second & $129,117(28 \%)$ & $4907(27 \%)$ & $8984(27 \%)$ \\
\hline Third & $128,213(27 \%)$ & $4810(26 \%)$ & $8683(26 \%)$ \\
\hline Fourth & $113,362(24 \%)$ & $4088(22 \%)$ & $7398(22 \%)$ \\
\hline \multicolumn{4}{|l|}{ Resident area } \\
\hline Metropolitan (large) & $215,430(46 \%)$ & $8872(49 \%)$ & $15,803(48 \%)$ \\
\hline Metropolitan (medium) & $102,357(22 \%)$ & $3715(20 \%)$ & $6865(21 \%)$ \\
\hline Metropolitan (small) & $54,121(12 \%)$ & $2119(12 \%)$ & $3731(11 \%)$ \\
\hline Micropolitan & $55,219(12 \%)$ & $2021(11 \%)$ & $3789(12 \%)$ \\
\hline Rural & $41,288(9 \%)$ & $1493(8 \%)$ & $2700(8 \%)$ \\
\hline \multicolumn{4}{|l|}{ Hospital size } \\
\hline Small & $93,133(20 \%)$ & $3236(19 \%)$ & $5844(18 \%)$ \\
\hline Medium & $118,551(25 \%)$ & $4774(26 \%)$ & $8652(26 \%)$ \\
\hline Large & $256,826(55 \%)$ & $10,215(56 \%)$ & $18,403(56 \%)$ \\
\hline \multicolumn{4}{|l|}{ Hospital type } \\
\hline Public & $44,000(9 \%)$ & $1994(11 \%)$ & $3492(11 \%)$ \\
\hline Nonprofit & $358,700(77 \%)$ & $13,298(73 \%)$ & $24,188(74 \%)$ \\
\hline Private & $65,810(14 \%)$ & $2933(16 \%)$ & $5220(16 \%)$ \\
\hline \multicolumn{4}{|l|}{ Teaching } \\
\hline Yes & $254,754(54 \%)$ & $10,044(55 \%)$ & $18,266(56 \%)$ \\
\hline No & $213,756(46 \%)$ & $8181(45 \%)$ & $14,634(44 \%)$ \\
\hline \multicolumn{4}{|l|}{ Payer } \\
\hline Medicare & $272,202(58 \%)$ & $12,335(68 \%)$ & $21,564(66 \%)$ \\
\hline Private & $160,470(34 \%)$ & $4289(24 \%)$ & $8437(26 \%)$ \\
\hline Medicaid & $14,960(3 \%)$ & $912(5 \%)$ & $1654(5 \%)$ \\
\hline Other & $20,878(4 \%)$ & $689(4 \%)$ & $1245(4 \%)$ \\
\hline
\end{tabular}

APR DRG = all patient-refined diagnosis-related group. 


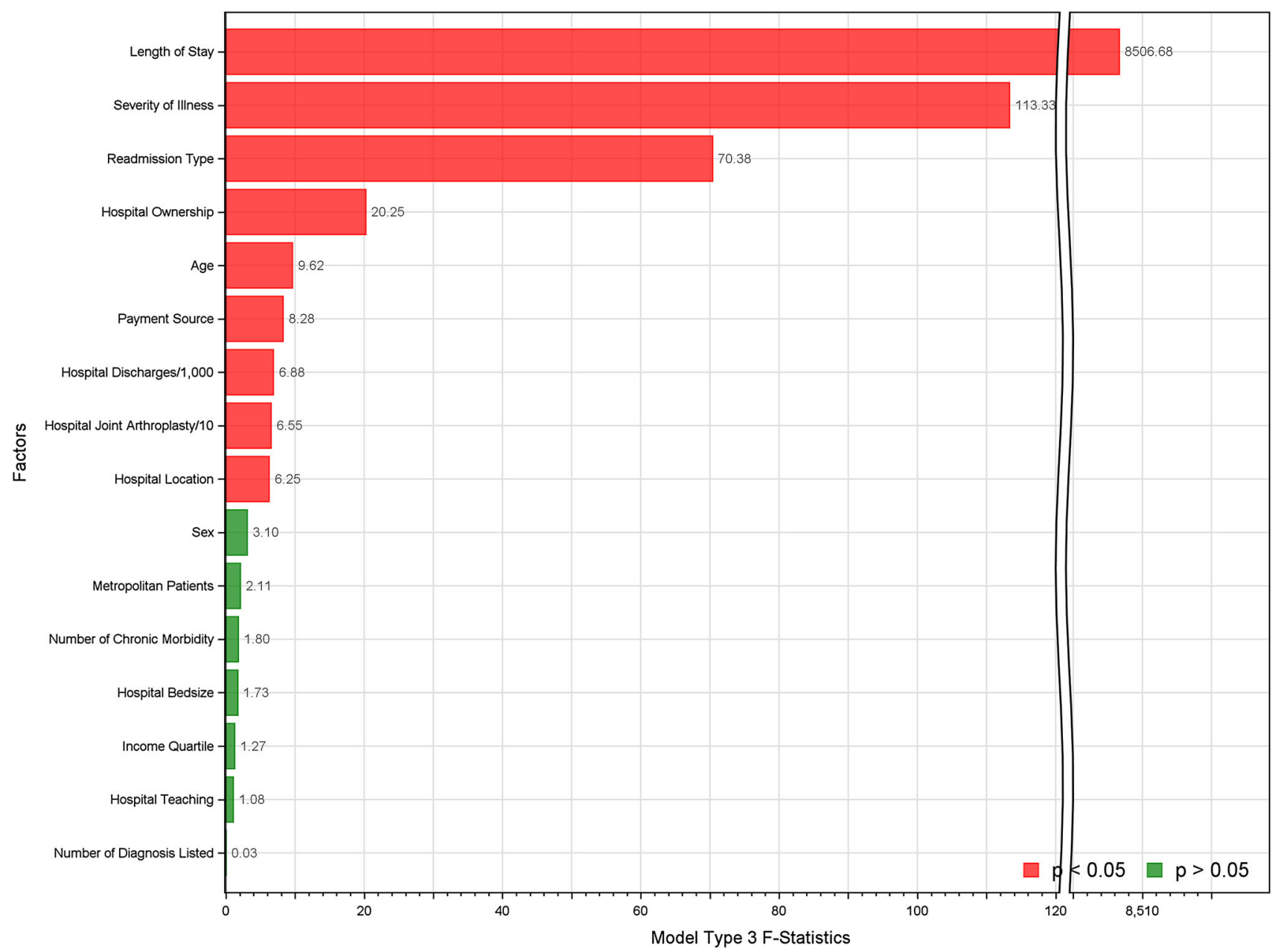

Fig. 1 Clinical and patient factors are more strongly associated than hospital factors with 90-day readmission costs after THA.

239,700 days of hospital stays. Overall, hospital readmissions after THA and TKA represent an annualized USD 1.1 billion economic burden on the US healthcare system.

\section{Most Costly Types of Readmissions}

Procedure-related complications (such as infection) were more common in the initial 30 days after THA and account for $70 \%(95 \% \mathrm{CI}, 69.5 \%-70.2 \%)$ of the total annual cost for the United States. In 90 days after THA, more readmissions were still associated with procedure-related complications, which account for 59\% (95\% CI, 59.1\%$59.6 \%$ ) of the total readmission costs in 90 days. The five most costly types of THA 90-day readmissions, in terms of their annual economic burden (Fig. 3), were infections (USD 50 million; 95\% CI, USD 43.0-56.9 million), dislocations (USD 36 million; 95\% CI, USD 31.2-40.9 million), periprosthetic fractures (USD 23 million; $95 \% \mathrm{CI}$, USD 19.7-26.9 million), issues associated with osteoarthrosis (USD 18 million; 95\% CI, USD 14.7-21.2 million), and other postoperative infections (USD 18 million; 95\% CI, USD 15.0-20.0 million). Infection of the joint prosthesis represented $6 \%$ of 90 -day readmissions, but accounted for $11 \%$ of the total readmission costs. Other conditions that also commanded a high national cost for readmission included hematoma (USD 11 million; 95\% CI, USD 9.4-13.2 million), acute cardiac events (USD 33 million; 95\% CI, USD 28.8-37.8 million), and acute vascular and thrombotic events (USD 13 million; 95\% CI, USD 10.6-15.4 million).

Similar to THA, the majority of the 30-day short-term readmission cost burden for TKA was associated with procedural complications (60\%; 95\% CI, 59.5\%-60.6\%), whereas $49 \%$ of the total readmission cost burden in 90 days was associated with procedure issues (95\% CI, $48.8 \%-49.6 \%$ ). The five most costly types of TKA 90-day readmissions, in terms of their annual economic burden (Fig. 4), were infections (USD 64 million; 95\% CI, USD 56.8-70.9 million), localized osteoarthrosis (USD 42 million; 95\% CI, USD 36.3-46.9 million), septicemia (USD 27 million; 95\% CI, USD 22.2-31.4 million), other 


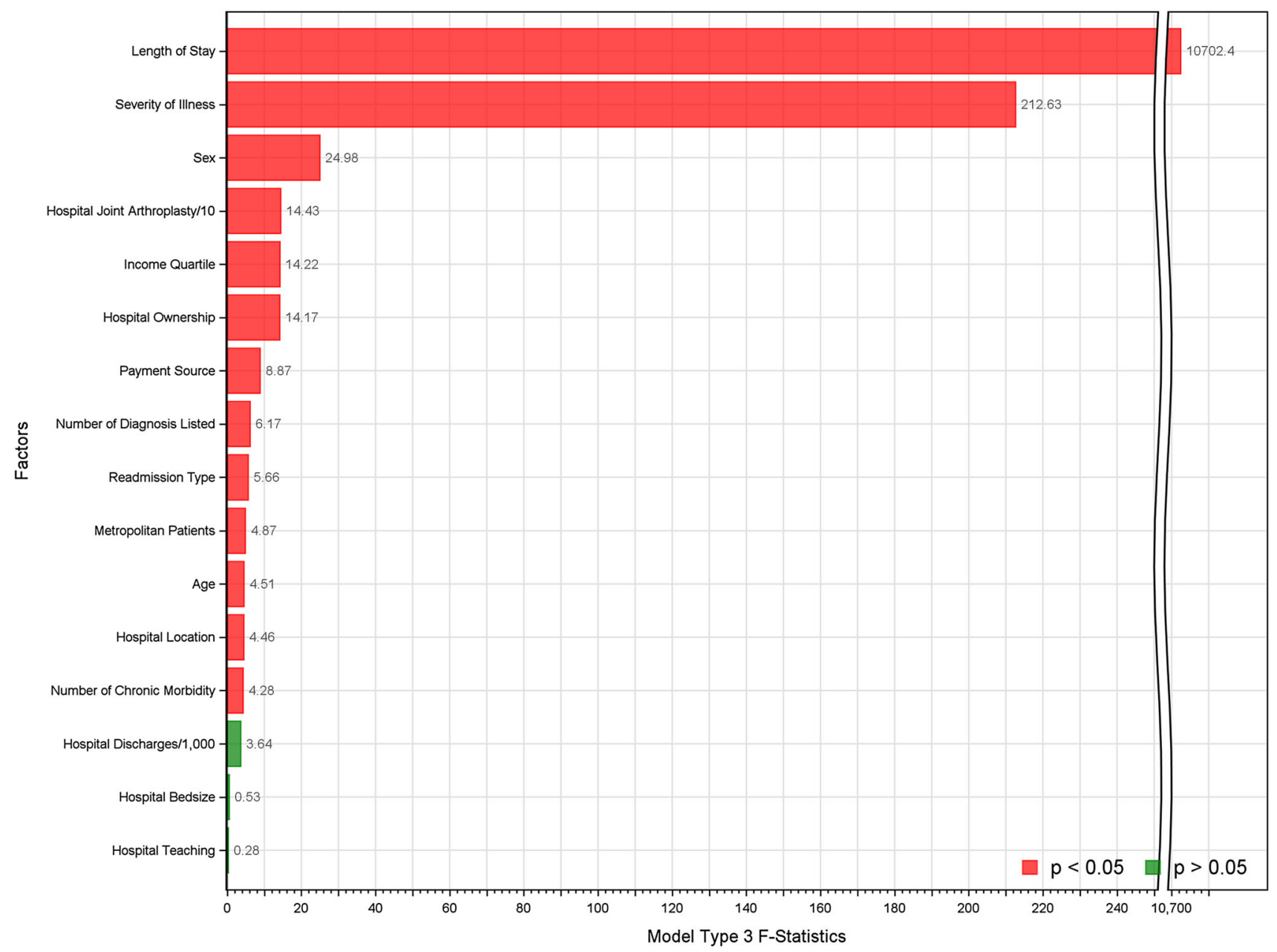

Fig. 2 Clinical and patient factors are more strongly associated than hospital factors with 90-day readmission costs after TKA.

postoperative infection (USD 25 million; 95\% CI, USD 21.5-27.5 million), and acute subendocardial infarction (USD 12 million; 95\% CI, USD 8.4-14.7 million). As a group, acute cardiac events (USD 52 million; 95\% CI, USD 46.3-57.5 million) and acute vascular and thrombotic events (USD 23 million; 95\% CI, USD 20.1-25.0 million) were also associated with a high total readmission cost.

\section{Discussion}

Although the ultimate destiny of the ACA is open to debate, many innovations of healthcare reform, including value-based episodes of care and bundled payments, are now so engrained by CMS and so widely adopted by the US healthcare marketplace that they will prove challenging to completely dispel in the near future. As a quality metric for orthopaedic surgery, hospital readmission has been studied previously to identify risk factors for stratifying patients in terms of their odds for readmission $[1,10,11,17,21,22,24,25,27,29,32,33,36-38]$. This metric varies depending on hospital or provider perspective. However, as orthopaedic surgeons increasingly become financial stakeholders in bundled payment models, the economic dimension of readmissions is important for clinicians to appreciate. The present study is the first to our knowledge that adopted a nationwide perspective on this issue using a new national database, the NRD. Using this database, we sought to determine which patient, hospital, and clinical factors were associated with the cost of readmissions for the US healthcare system. For both THA and TKA, we found that LOS and APR DRG severity were the most important predictors of the cost for a readmission episode. Not only did we find that 90-day readmissions occur more frequently among Medicare beneficiaries, but we also determined that almost two-thirds of the total national cost for readmissions is reimbursed by Medicare. The most costly types of procedure-related readmissions, in terms of their total national cost to the US healthcare system, were the result of infection (this applied to both THA and TKA), dislocation (THA only), and periprosthetic fracture (THA only). 


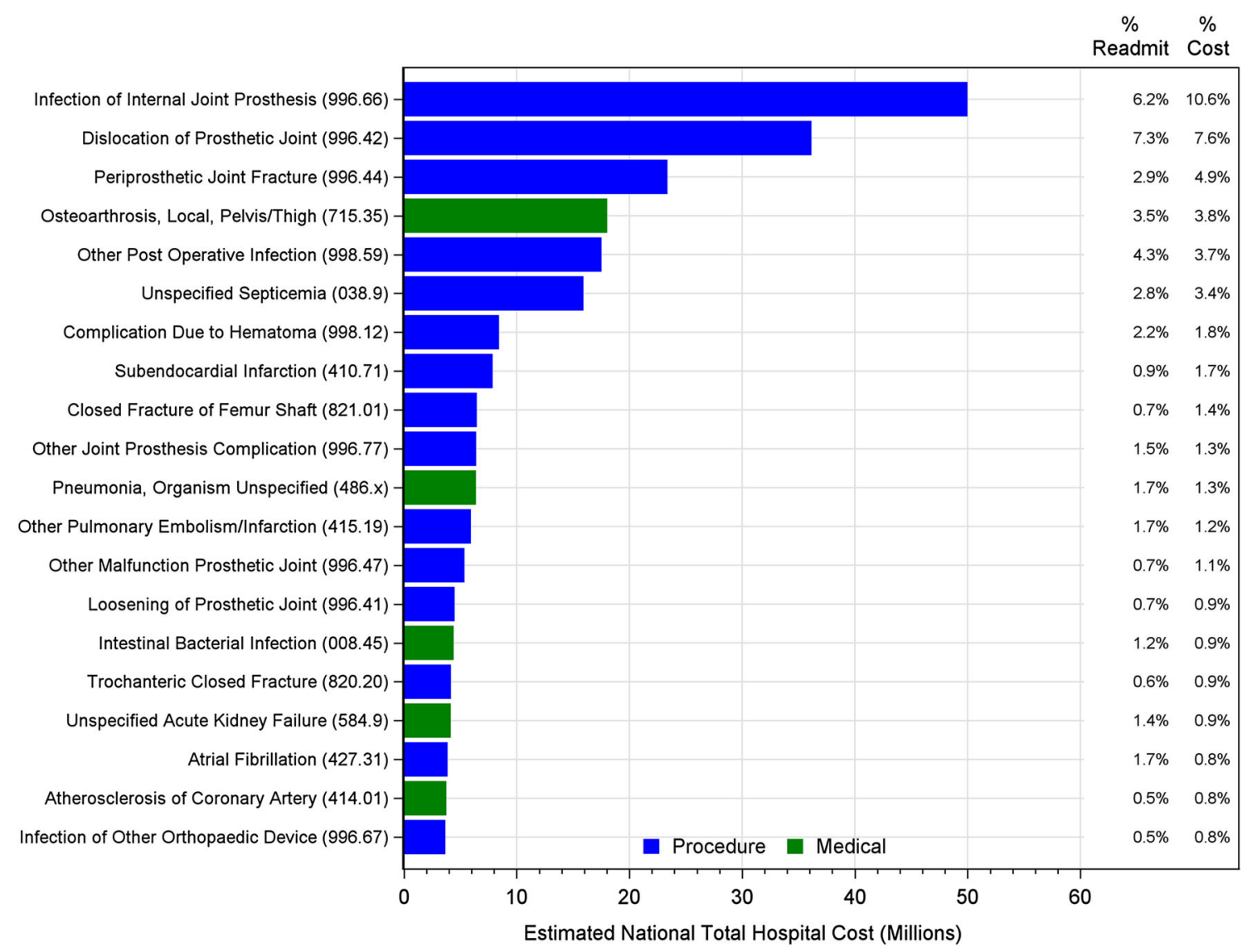

Fig. 3 The most costly 90-day readmissions after THA, in terms of their total national cost, are procedure-related.

Our study has several important limitations. First, we calculated procedure and readmission costs from hospital charges by using a cost-to-charge ratio for each hospital in the NRD provided by the HCUP. In recent research on this topic, cost has been defined using a variety of metrics, including the approach we used in this study (cost-tocharge ratios) [6]. Others have used hospital charges, claims, or billing data $[31,34]$; payments or reimbursements $[5,8,26]$ as well as the measure of direct costs from an individual hospital's cost-accounting system $[3,8,9,30]$. An individual hospital's charges and internal cost accounting data are not generalizable across the entire United States. In other words, using the cost-to-charge ratio as we have done here allows us to compare "costs" across geographic areas, but may not really reflect "true costs" in a way that a provider or hospital might understand the term. True cost is very hard to estimate at a single institution let alone across the entire country. Thus, using payment information as a surrogate for cost implies a societal or payer perspective as opposed to a hospital perspective to an economic analysis. For the present study, we adopted the same hospital perspective-based cost metric used by not only CMS, but also all of the HCUP databases made publicly available by the US Department of Health and Human Services. These disparities in cost metrics make it challenging to quantitatively compare our results with previous studies on the topic.

Second, we limited the analysis to in-state patients because the NRD is limited to in-state readmissions. We validated the NRD data by using the $100 \%$ Medicare inpatient database for 2013 to determine the loss to followup across state boundaries. We found that $95 \%$ or more of readmissions occurred within the same state as the primary arthroplasty and therefore concluded that the statebased sampling plan for the NRD does not undermine the conclusions of our study.

Third, we classified readmissions as procedure- or medical-related based on their ICD-9-CM diagnosis codes. Whereas some diagnosis codes such as deep infection can reasonably be attributed to primary surgery within 90 days, other diagnoses such as septicemia may be arguably more ambiguous to attribute without access to the patient's full medical records. We classified some of these more ambiguous codes as procedure-related (as detailed in Appendix 1). Even with this worst case classification scenario, medical-related diagnoses were associated with $41 \%$ to $51 \%$ of the economic burden for 90-day readmissions. Fourth, we analyzed inpatient costs in the current analysis, 


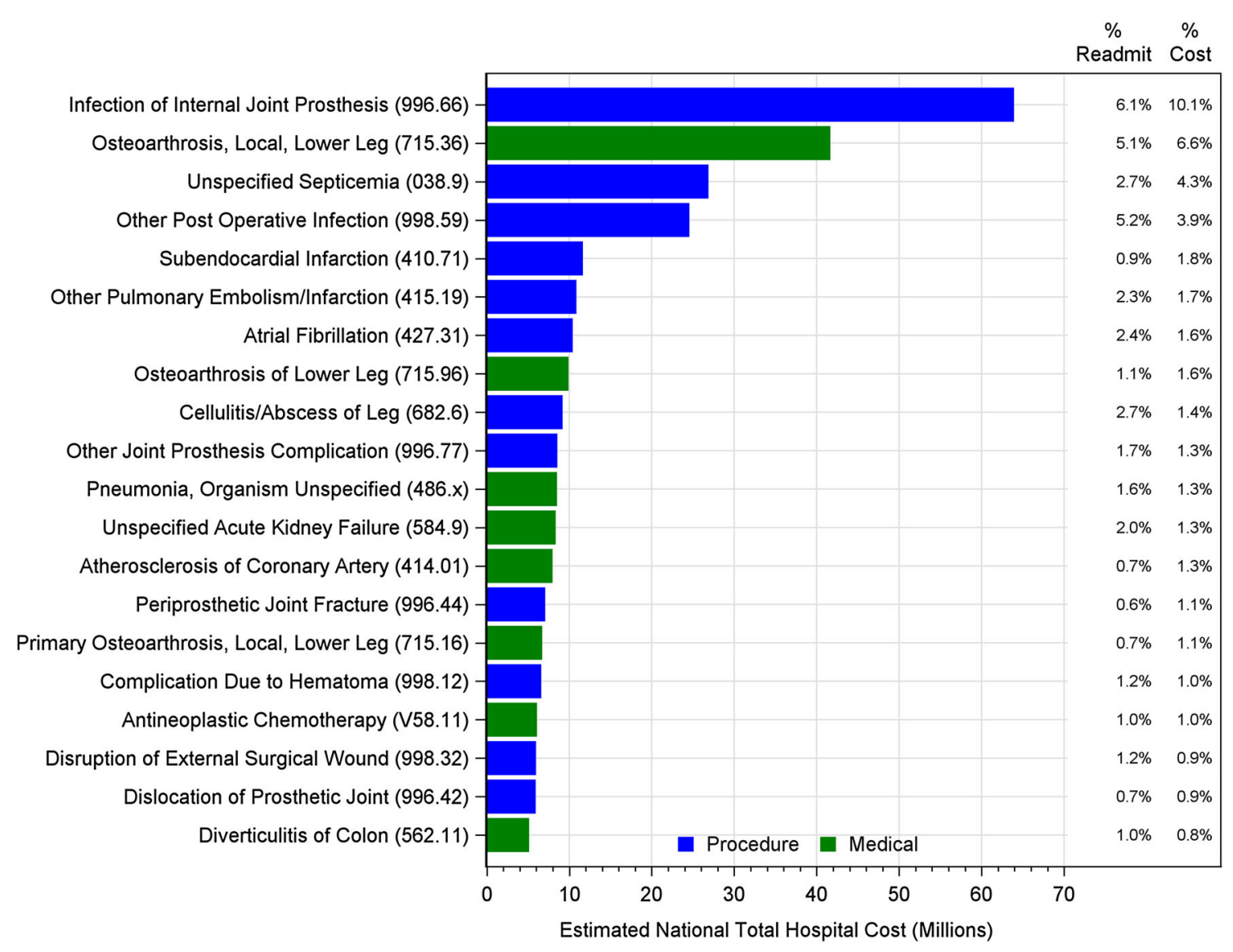

Fig. 4 The most costly 90-day readmissions after TKA, in terms of their total national cost, are procedure-related.

and thus the economic burden did not include any outpatient or professional (physician) costs for treating these readmitted patients.

The national readmission rates for THA and TKA are consistent with the findings of others $[1,10,11,17,21$, $22,24,25,27,29,32,33,36-38]$ and are lower than the readmission rates reported for the Medicare population. For example, at 90 days, the national readmission rates in the present study for THA and TKA are $8 \%$ and $7 \%$, respectively. These rates are lower than the previously reported 90-day readmissions rates for Medicare beneficiaries [21, 22], which corresponded to $12.7 \%$ and $9.6 \%$ for THA and TKA, respectively. We found that readmissions occur much less frequently in the private patient population (Tables 1,2), which may be explained because patients undergoing arthroplasty who are covered by private insurance tend to be younger and healthier than patients on Medicare [6, 31]. Thus, the lower incidence of readmission among the private paying arthroplasty population tends to drive down the national readmission rates, below what one would expect solely by focusing on the Medicare population in isolation. Previous researchers have examined arthroplasty readmissions in a variety of subpopulations in the United States $[1,10,11,17,21,22,24,25,27,29$, $32,33,36-38]$. However, the present study is the first of which we are aware to survey the readmission rates from a national perspective, including all payers and patient age groups.

Relatively few previous researchers have examined the patient, hospital, and clinical factors associated with the cost of arthroplasty readmissions [6, 31]. Plate and colleagues [31] studied the patient factors associated with readmission cost at their hospital and found that the reasons for readmission were a predictor of costs. Chan et al. [6] studied factors related to readmission cost of TKA in the state of Michigan using the SID, also provided by HCUP. The predictors of TKA readmission cost in Chan et al.'s study [6] included LOS, discharge disposition, number of chronic conditions, and the total cost of the initial admission. Consistent with Chan et al.'s work, we also observed that the LOS during the readmission was most strongly associated with cost. For primary arthroplasty, the LOS has been decreasing over time in an effort to contain costs $[10,11]$; a similar strategy is likely to be effective for reducing the costs of readmissions. There comes a time, however, when decreasing LOS is no longer practically 
feasible and simultaneously maintains quality of care; a multipronged approach to readmission cost control is therefore warranted.

To our knowledge, this study is the first to quantify the national economic burden of arthroplasty readmissions by different payers. Previous researchers $[6,31]$ have examined whether an individual patient's readmission costs varied by payer. Chan et al. [6], in their analysis of 1721 readmitted patients in Michigan in 2012, considered payers as a factor in their statistical analysis of costs. Chan et al. did not report a difference between the readmission costs of "Medicare-or-Medicaid" versus "non-Medicare-nor-Medicaid" groups of patients. Plate and colleagues [31], in their single-institution study of THA readmissions, likewise found that the readmission costs for Medicare/Medicaid and private payer patients were "similar." Because the sample size available from the NRD was much larger than in previous studies, we were able to detect a relatively modest difference in cost between Medicare and other payers of readmissions using a multivariate model that accounted for patient, hospital, and clinical factors. The results of this study therefore underscore the importance of focusing on reducing the readmission burden for the Medicare population. In light of available evidence, it seems reasonable that efforts to mitigate the readmission burden for patients on Medicare will likewise translate to improvements in the care for patients reimbursed outside of Medicare. We found the NRD to be useful for the present study, but this new data set is by no means necessary to investigate the greatest THA and TKA populations at risk for readmission and their associated economic burden. For other orthopaedic and spine procedures, which treat a greater proportion of younger patients, the NRD may prove to be more indispensable as a research tool.

Our study adds to the growing body of work examining the economic burden for specific types of readmissions $[5,8,31]$. Our findings agree with previous researchers that infections are among the most costly $[8,31]$. Our findings are also consistent with earlier research that identified the costs for medical readmissions [8]. The most costly readmissions were generally related to medical complications and unrelated to the total joint arthroplasty itself. In light of the substantial economic burden of medical readmissions, it remains open to debate whether it is reasonable for these unrelated costs to be borne by the hospital or bundle provider when they have no causal relationship to the arthroplasty. That said, some $50 \%$ of readmissions at 90 days appear to be related to issues with the procedure itself. This is an area where physicians and hospitals can consider strategies to reduce readmission costs directly. In contrast with previous studies, we quantified the national economic burden for specific types of readmissions. Our findings will be useful to hospitals, payment bundle providers, and policymakers seeking to target efforts to reduce the economic burden of readmissions.

This national study underscores LOS during readmission as a primary cost driver, suggesting that hospitals and doctors further optimize, to the extent possible, the clinical pathways for the hospitalization of readmitted patients. Because not all readmissions are equally expensive, this study identifies several areas on which members of the orthopaedic community can focus attention and thereby hopefully strategically reduce the economic impact of readmissions. Because patients readmitted because of infection, dislocation, and periprosthetic fractures are the most costly types of readmissions, efforts to reduce the LOS for these types of readmissions will have the greatest impact on their economic burden. It is debatable whether LOS is in fact modifiable or merely reflects the inherent comorbidity mix of the readmitted patients. Physicians and clinical care coordinators may have an effect on LOS by addressing the issue during preoperative consultation. Even when LOS expectations are addressed preoperatively, certain postoperative factors including pain management, complications, ambulatory ability, and family support may result in an extended hospital stay. Severity of illness is dependent on the patient's underlying problems and not modifiable. Thus, it remains to be seen whether LOS during the readmission episode after THA or TKA is, in fact, modifiable to any economically meaningful extent. Additional clinical research is needed to determine the extent to which, if any, the LOS during readmissions can be reduced without sacrificing quality or access of care.

\section{References}

1. Avram V, Petruccelli D, Winemaker M, de Beer J. Total joint arthroplasty readmission rates and reasons for 30-day hospital readmission. J Arthroplasty. 2014;29:465-468.

2. Boozary AS, Manchin J 3rd, Wicker RF. The Medicare hospital readmissions reduction program: time for reform. JAMA. 2015;314:347-348.

3. Bosco JA 3rd, Karkenny AJ, Hutzler LH, Slover JD, Iorio R. Cost burden of 30-day readmissions following Medicare total hip and knee arthroplasty. J Arthroplasty. 2014;29:903-905.

4. Bozic KJ, Chiu V. Quality measurement and public reporting in total joint arthroplasty. J Arthroplasty. 2008;23:146-149.

5. Bozic KJ, Ward L, Vail TP, Maze M. Bundled payments in total joint arthroplasty: targeting opportunities for quality improvement and cost reduction. Clin Orthop Relat Res. 2014;472:188193.

6. Chan MY, Malik SA, Hallstrom BR, Hughes RE. Factors affecting readmission cost after primary total knee arthroplasty in Michigan. J Arthroplasty. 2016;31:1179-1182.

7. Christo AE, Bargar WL, Morris E. Prosthesis cost containment in total joint replacement: a physician-driven free-market approach. Orthopedics. 2000;23:439-442.

8. Clair AJ, Evangelista PJ, Lajam CM, Slover JD, Bosco JA, Iorio R. Cost analysis of total joint arthroplasty readmissions in a 
bundled payment care improvement initiative. J Arthroplasty. 2016;31:1862-1865.

9. Clement RC, Kheir MM, Derman PB, Flynn DN, Speck RM, Levin LS, Fleisher LA. What are the economic consequences of unplanned readmissions after TKA? Clin Orthop Relat Res. 2014;472:3134-3141.

10. Cram P, Lu X, Kaboli PJ, Vaughan-Sarrazin MS, Cai X, Wolf BR, Li Y. Clinical characteristics and outcomes of Medicare patients undergoing total hip arthroplasty, 1991-2008. JAMA. 2011;305:1560-1567.

11. Cram P, Lu X, Kates SL, Singh JA, Li Y, Wolf BR. Total knee arthroplasty volume, utilization, and outcomes among Medicare beneficiaries, 1991-2010. JAMA. 2012;308:1227-1236.

12. Filson CP, Hollingsworth JM, Skolarus TA, Clemens JQ, Hollenbeck BK. Health care reform in 2010: transforming the delivery system to improve quality of care. World J Urol. 2011;29:85-90.

13. Garber A, Goldman DP, Jena AB. The promise of health care cost containment. Health Aff (Millwood). 2007;26:1545-1547.

14. Iorio R. Strategies and tactics for successful implementation of bundled payments: bundled payment for care improvement at a large, urban, academic medical center. J Arthroplasty. 2015;30:349-350.

15. Iorio R, Healy WL, Richards JA. Comparison of the hospital cost of primary and revision total knee arthroplasty after cost containment. Orthopedics. 1999;22:195-199.

16. James J. Health policy brief: Medicare hospital readmissions reduction program. Health Aff (Milwood). 2013 Nov 12. [Epub ahead of print]

17. Jordan CJ, Goldstein RY, Michels RF, Hutzler L, Slover JD, Bosco JA 3rd. Comprehensive program reduces hospital readmission rates after total joint arthroplasty. Am J Orthop (Belle Mead NJ). 2012;41:E147-151.

18. Keehan SP, Cuckler GA, Sisko AM, Madison AJ, Smith SD, Stone DA, Poisal JA, Wolfe CJ, Lizonitz JM. National health expenditure projections, 2014-24: spending growth faster than recent trends. Health Aff (Millwood). 2015;34:1407-1417.

19. Keswani A, Uhler LM, Bozic KJ. What quality metrics is my hospital being evaluated on and what are the consequences? $J$ Arthroplasty. 2016;31:1139-1143.

20. Kondo K. Beyond the era of cost containment. Jpn Hosp. 2008:19-38.

21. Kurtz SM, Lau EC, Ong KL, Adler EM, Kolisek FR, Manley MT. Hospital, patient, and clinical factors influence 30- and 90-day readmission after primary total hip arthroplasty. $J$ Arthroplasty. 2016;31:2130-2138.

22. Kurtz SM, Lau EC, Ong KL, Adler EM, Kolisek FR, Manley MT. Which hospital and clinical factors drive 30- and 90-day readmission after TKA? J Arthroplasty. 2016;31:2099-2107.

23. Kurtz SM, Ong KL, Lau E, Bozic KJ. Impact of the economic downturn on total joint replacement demand in the United States: updated projections to 2021. J Bone Joint Surg Am. 2014;96:624-630.

24. Lavernia CJ, Villa JM. Readmission rates in total hip arthroplasty: a granular analysis? J Arthroplasty. 2015;30:11271131.
25. Mesko NW, Bachmann KR, Kovacevic D, LoGrasso ME, O'Rourke C, Froimson MI. Thirty-day readmission following total hip and knee arthroplasty-a preliminary single institution predictive model. J Arthroplasty. 2014;29:1532-1538.

26. Nichols CI, Vose JG. Clinical outcomes and costs within 90 days of primary or revision total joint arthroplasty. J Arthroplasty. 2016;31:1400-1406.e1403.

27. Oronce CI, Shao H, Shi L. Disparities in 30-day readmissions after total hip arthroplasty. Med Care. 2015;53:924-930.

28. Orszag PR. US health care reform: cost containment and improvement in quality. JAMA. 2016;316:493-495.

29. Paxton EW, Inacio MC, Singh JA, Love R, Bini SA, Namba RS. Are there modifiable risk factors for hospital readmission after total hip arthroplasty in a US healthcare system? Clin Orthop Relat Res. 2015;473:3446-3455.

30. Peel TN, Cheng AC, Liew D, Buising KL, Lisik J, Carroll KA, Choong PF, Dowsey MM. Direct hospital cost determinants following hip and knee arthroplasty. Arthritis Care Res. 2015;67:782-790.

31. Plate JF, Brown ML, Wohler AD, Seyler TM, Lang JE. Patient factors and cost associated with 90-day readmission following total hip arthroplasty. J Arthroplasty. 2016;31:49-52.

32. Pugely AJ, Callaghan JJ, Martin CT, Cram P, Gao Y. Incidence of and risk factors for 30-day readmission following elective primary total joint arthroplasty: analysis from the ACS-NSQIP. $J$ Arthroplasty. 2013;28:1499-1504.

33. Raines BT, Ponce BA, Reed RD, Richman JS, Hawn MT. Hospital acquired conditions are the strongest predictor for early readmission: an analysis of 26,710 arthroplasties. $J$ Arthroplasty. 2015;30:1299-1307.

34. Ramos NL, Wang EL, Karia RJ, Hutzler LH, Lajam CM, Bosco JA. Correlation between physician specific discharge costs, LOS, and 30-day readmission rates: an analysis of 1,831 cases. $J$ Arthroplasty. 2014;29:1717-1722.

35. Readmissions Reduction Program (HRRP). Available at: https:// www.cms.gov/medicare/medicare-fee-for-service-payment/acute inpatientpps/readmissions-reduction-program.html. Accessed December 28, 2016.

36. Saucedo JM, Marecek GS, Wanke TR, Lee J, Stulberg SD, Puri L. Understanding readmission after primary total hip and knee arthroplasty: who's at risk? J Arthroplasty. 2014;29:256-260.

37. Schairer WW, Vail TP, Bozic KJ. What are the rates and causes of hospital readmission after total knee arthroplasty? Clin Orthop Relat Res. 2014;472:181-187.

38. Singh JA, Inacio MC, Namba RS, Paxton EW. Rheumatoid arthritis is associated with higher ninety-day hospital readmission rates compared to osteoarthritis after hip or knee arthroplasty: a cohort study. Arthritis Care Res. 2015;67:718-724.

39. Stabile M, Thomson S, Allin S, Boyle S, Busse R, Chevreul K, Marchildon G, Mossialos E. Health care cost containment strategies used in four other high-income countries hold lessons for the United States. Health Aff (Millwood). 2013;32:643-652. 